\title{
Research on the Impact of Ultimate Ownership Structure on Corporate Social Responsibility
}

\author{
Su Kun ${ }^{1, a}$, Yuan An-qi2,b, \\ ${ }^{1}$ Department of accounting, Northwestern Polytechnical University, Xi'an, Shaanxi, China \\ ${ }^{2}$ Department of accounting, Northwestern Polytechnical University, Xi'an, Shaanxi, China \\ asukun@nwpu.edu.cn, ${ }^{b} 1561989424 @ q q . c o m$ \\ *Yuan An-qi
}

Keywords : Ultimate ownership structure; Pyramid level; Corporate Social Responsibility; Government level

\begin{abstract}
Based on the data of Shanghai and Shenzhen A-share listed companies from 2011 to 2017, this paper analyzes the ultimate ownership structure and pyramid level, and studies the impact of actual controllers on the corporate social responsibility of listed companies. The empirical results show that when corporate social responsibility as an agent cost, with the increase of cash flow right, the initiative of the actual controller to undertake corporate social responsibility decreases, and the cash flow right and corporate social responsibility are significantly negatively correlated. And because of the separation of cash flow right and control right, the actual controller often takes corporate social responsibility as a tool to confuse the public vision, and the degree of separation of the two rights and the pyramid level have a significant positive correlation with corporate social responsibility. At the same time, this study also found that compared with non-state-owned enterprises, state-owned enterprises, as the embodiment of the will of the state, bear more corporate social responsibility. With the reduction of the level of government, the positive effect of pyramid level on corporate social responsibility is weakening.
\end{abstract}

\section{终极所有权结构对企业社会责任的影响研究 \\ 苏坤 ${ }^{1, a}$, 元安琪 $2, b^{*}$ \\ 1西北工业大学会计系, 西安, 陕西, 中国 \\ 2西北工业大学会计系, 西安, 陕西, 中国 \\ asukun@nwpu.edu.cn, b1561989424@qq.com \\ *元安琪}

关键词: 终极所有权结构; 金字塔层级; 企业社会责任; 政府级别

中文摘要. 本文以我国沪深 A 股上市公司 2011-2017 年数据为样本, 通过分析终极所有权结 构、金字塔层级, 研究实际控制人对上市公司承担企业社会责任的影响。实证研究结果显示, 当企业社会责任作为一种代理成本时，随着现金流权的增加，实际控制人承担企业社会责任 的主动性降低, 现金流权与企业社会责任呈显著负相关关系, 并且由于现金流权与控制权的 分离, 实际控制人为掩盖 “掏空” 等不当行为, 往往会将企业社会责任作为混淆公众视野的 工具, 两权分离度和金字塔层级数与企业社会责任呈显著正相关关系。同时本研究还发现, 相对于非国有企业, 国有企业作为国家意志的体现，承担着更多企业社会责任，伴随着政府 级别的降低，金字塔层级对企业承担社会责任的正向作用在减弱。 


\section{1. 引言}

随着各领域学者对企业社会责任不断深入的研究和推广, 中国企业在承担社会责任方面 有了长足的进步和发展。根据《金蜜蜂企业社会责任蓝皮书 2018》公布的数据显示, 从 2001 年至 2018 年, 中国企业社会责任报告数量逐年增长。截至 2018 年 10 月 31 日, 中国社会责 任报告一共发布了 1676 份, 披露也更加完善和系统。

但是上述披露行为是否能真正促进企业的可持续发展? 剥离现有的表面现象，我们发现 企业在公司内部治理方面所做的改变并非实质性。一方面, 我国企业社会责任报告存在着大 量的强制性披露, 而自愿披露的数量明显偏低（权小峰等，2015） [1]，这可能是出于我国产 权保护不完善以及政府干预等因素的影响, 企业自愿披露信息的积极性较差。另一方面, 企 业社会责任报告数量的上涨并没有带动其质量的提升, 在相当长的一段时间内, 企业社会责 任报告仍然被认为是公关的一种发明, 企业将其作为锦上添花的门面装饰, 甚至说, 企业社 会责任报告谁编谁看, 利益相关者不能从中获得真正能反映企业实际状况的信息。之前对企 业社会责任的研究主要是基于企业社会责任对企业形象的积极正面影响, 而本文则将以我国 企业社会责任承担的现实状况进行深入研究。并且, 在之前的研究中, 学者们主要是从股权 结构的角度出发探究股东和管理层之间的代理问题对企业社会责任的影响, 但没有将终极控 股股东这个因素考虑进来, 即对第二类代理问题对企业社会责任的影响少有研究, 这对研究 股权结构与企业社会责任之间的关系并不能起到积极的推动作用。

金字塔持股结构现如今被越来越多的企业使用，在中国当前的市场环境下，不论是国有 企业还是非国有企业，其公司股权结构中都存在着金字塔股权结构。进一步研究可以发现， 国有企业与非国有企业建立金字塔股权结构的动机也存在着较大差异, 国有企业的初衷是为 简政放权提供便利从而实行该控股方式, 而非国有企业则是为了通过金字塔股权结构挸取公 司利益、谋求个人私利。中国作为一个正在崛起的发展中国家, 政府对市场资源配置起主导 性作用，而市场资源是企业赖以生存的基础，这使得企业之间建立政治关联的积极性不断增 强, 企业社会责任作为树立企业形象、增强外部环境稳定性、建立企业与政府之间的桥梁和 纽带的重要工具, 被越来越多的企业所接受, 终极控股股东为了实现企业形象和价值的双赢 结果，将承担企业社会责任作为重要的公司战略，向市场传递有利于公司的市场信息。

从上述讨论出发, 本文以 2011-2017 年中国上市公司为研究对象, 探究现金流权和两权 分离度对企业社会责任的影响, 并从金字塔持股结构多层级的结构特征出发, 剖析其层级数 的多少对企业社会责任的影响，比较不同产权性质的上市公司承担企业社会责任的差异性， 并进一步的揭示在国有上市公司中不同的政府行政级别在金字塔层级对企业社会责任影响的 调节作用。

\section{2. 理论分析与研究假设}

纵观中国社会经济和产业集群的发展与沿革，区域经济的协调发展对企业社会责任的期 望变得愈加强烈, 企业作为集群内经济行为的促发者和执行者, 其社会责任的主体作用日益 凸显。企业社会责任对企业经济行为和市场发展的影响一直是学术界争论的焦点问题。认清 企业社会责任首先需要理解企业从事履行社会责任的原因。Derwall 等（2011） ${ }^{[2]}$ 提出企业社 会责任建设受到价值驱动和机会驱动两种动机的影响。价值驱动假说认为提升企业价值, 实 现股东和利益相关方利益的一致是企业管理者履行社会责任的动因, 企业社会责任不仅是一 种伦理道德的要求, 作为社会的一部分, 企业履行社会责任也是一种社会需求。企业社会责 任满足了供应商、消费者、投资者等利益相关方的需求, 形成了维护各个关系基础的无形资 产, 提升公司融资能力以及应对风险的水平, 有助于降低管理层进行盈余管理的可能性。总 之, 价值驱动假说将企业社会责任视为提升企业价值、树立企业良好形象的积极驱动因素。 在机会推动假说的前提下, 企业社会责任更多体现了其工具属性而非价值属性, 管理层 
履行企业社会责任是为实现个人利益最大化、提升个人职业声誉等目的。Hemingway 和 Maclagan（2004） [3]研究发现，公司发布企业社会责任报告的目的是掩盖某些不当行为，维 护公司良好的外部形象。权小锋等 (2015) [1]发现企业社会责任作为管理层隐瞒坏消息以及 转移股东审查的工具, 会恶化企业的信息透明度并强化管理层的捂盘行为, 造成股价崩盘风 险的加剧。程小可 (2013) ${ }^{[4]}$ 提出企业社会责任信息披露已经成为上市公司掩盖盈余管理的 工具, 当中国企业社会责任承担水平越高时, 㭆命管理水平也会越高, 这说明在中国, 企业 社会责任并不是企业 “道德意识” 的完全体现, 其本身就是一种代理成本, 不仅不能使股东 和利益相关方的利益趋于统一，反而会导致两者之间的分歧和企业资源的浪费，损害企业价 值。根据前述对我国企业社会责任的现状分析, 虽然承担企业社会责任得到不断地推广和实 施, 但就其反馈的结果来看我国企业更多的将履行企业社会责任作为强制性披露的义务, 并 且存在着较大可能性将企业社会责任作为粉饰公司形象的工具。

Shleifer 和 Vishny（1986） [5]通过模型发现很多发达国家的大企业中普遍存在着股权相对 集中的现象, 股权的相对集中使最突出的代理矛盾由股东和管理层之间转换到实际控制人和 广大中小股东之间。当实际控制人拥有的现金流权增加时，这意味着他依据现金流权分得的 利益更多, 其实现目标上市公司价值最大化的动机会更加强烈（La Porta 等，1999） [6]，掠夺 中小股东利益行为的倾向就会减弱 (Shleifer 和 Vishny，1997） [7]，利益趋同效应逐渐提升。 并且 Ingram（1983） [8]等研究发现在同等条件下, 企业承担社会责任产生的成本会使企业被 动的承担因市场波动、外部环境变化带来的损失, 削弱自身竞争实力, 此时, 企业社会责任 作为一种代理成本, 其承担的内部动机就会降低。在现金流权不断增加的情况下, 有限理性 的实际控制人为了其经济利益或者股东财富的最大化而将其应承担的成本转嫁给其他利益相

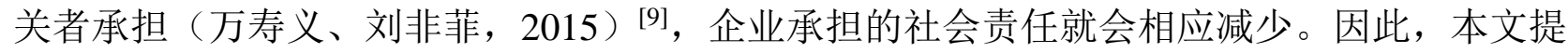
出如下假设:

假设 1：现金流权与企业社会责任负相关。

许多上市公司的终极控股股东通过金字塔结构、交叉持股与董事兼任等方式控制公司， 并造成控制权与现金流权的偏离（La Porta 等，1999）[6]。随着实际控制人控制权的增加，较 高的控制权比例增强了控股股东对上市公司的控制能力以及通过控制权占取私人收益的能 力。在这种情况下，外部股东等其他利益相关者的利益无法得到保证，这也就是所谓的“壕沟 效应”。当控制权比例不断提升时，实际控制人的收益与风险产生了明显的不对等，其利用少 量资源即可实现对公司收益的掠夺，这使公司绩效不稳定性增强。Yves 和 Richard (2007) [10] 研究发现控股股东往往会通过削弱公司内部控制机制的方式实现从中小股东身上篗取更多利 益, 两权分离程度越明显, 公司治理的有效性会越差。从上述分析中我们可以看出, 当企业 的两权分离度水平越高时, 实际控制人通过其较高的控制权比例占取私人利益, 此时为了减 少掏空行为对公司经营绩效的影响, 保证企业长期稳定运行, 实际控制人会通过承担企业社 会责任的方式转移公众的关注点（Hemingway 和 Maclagan，2004） [3]，此时实际控制人存在 美化公司形象的动机，更可能试图通过企业外部表现误导利益相关者，实际控制人的 “掏空” 行为越严重，承担社会责任的积极性越强。在此基础上，本文提出如下假设:

假设 2：现金流权与控制权的分离程度与企业社会责任承担正相关。

金字塔层级数是对公司控股结构纵向上的划分，它代表着终极控股股东与目标公司之间 的距离, 也是导致现金流权和控制权分离的重要原因（Claessens 等，2000） [11]。在金字塔股

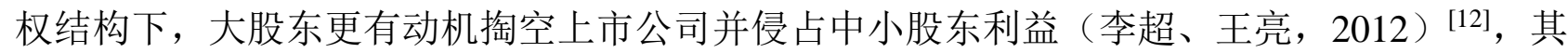
方式可能是通过过度投资或投资不足等非效率投资进行（刘星、窦炜 2009） [13]。股权相对集

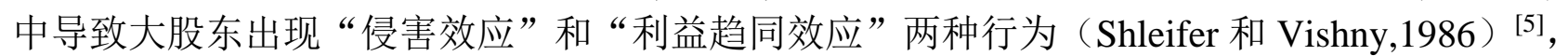
但不论是 “掏空” 公司价值、损害中小股东利益, 还是 “支持” 管理层行为、对管理层实施 有效监督，这两者之间都是密不可分的，大股东的 “掏空一支持一再掏空一再支持” 是一个 循环的过程, 现在的支持是为了未来的掏空 (陆宇建, 2002) [14], 但作为一个经济人, 大股 
东最终的目的还是实现掏空、谋求更多个人收益。为了 “保证大股东自身的利益, 改善公司 形象, 巩固品牌价值, 甚至是提高股价”，实际控制人往往选择成为 “负责任” 的企业 (Porter

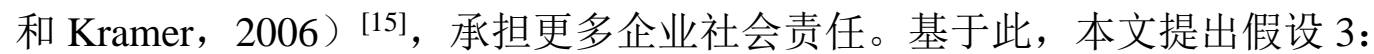

假设 3: 金字塔层级数与企业社会责任正相关。

不论在国有企业还是非国有企业中，都普遍存在着金字塔持股结构，但产权性质的不同 使非国有企业和国有企业在履行社会责任方面存在着较大差异（田志龙、贺远琼，2005） [16]。 国有企业作为政府的形象代言人，在社会责任方面起着 “榜样” 的作用，其经营决策往往与

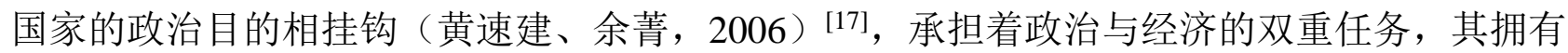
着制度严格、办事流程完善、以国家利益为导向的特点, 国有上市公司的许多行为体现更多 的是国家意志, 面临的问题也往往是民生福祉、国家安全的问题, 这在很大程度上决定了国 有上市公司承担着更多的社会责任, 这也是国有企业的特殊性所在。而对于非国有企业而言, 其实际控制人往往为个人或者家族, 个人利益与企业利益密切联系, 企业经营的目标就是追 求股东财富最大化, 遵循着成本收益原则, 企业社会责任作为一种 “超额成本” , 非国有企 业承担的意愿会相对减少。通过上述讨论，本文提出假设 4 。

假设 4：终极控制人为国有的上市公司承担更多的企业社会责任

为满足经济持续健康发展的迫切需求, “转变政府经济职能” 是当前形势下稳增长、控 通胀、防风险的重大举措。因此, 在前一项研究的基础上, 我们将国有企业作进一步的细分, 国有企业有省级、市级、县级的不同, 不同的政府行政级别会在金字塔层级数和链条数对经 营业绩的作用中产生差异 (张大勇, 2010) [18]研究发现, 较高的政府层级会加剧控制链条数 对公司经营业绩的负相关关系。我国行使的行政分权体制使各级政府的 “财”与与权” 有着 很大的不同, 也影响了各级政府对所控制国有企业的干预程度（苏坤，2015） [19]。对于高级 别政府而言, 其面临着更多的社会问题, 如基础设施建设、就业问题、国际局势等, 因而在 这个层面控制的企业受到国家意志的影响更显著, 会将社会效应而非经济效应放在首位。但 是, 对于市、县级政府而言, 其所控制的企业往往是实现当地经济发展目标、提高税收水平 的方式, 政府会减少对这类企业的干预从而保证企业的良好发展。据此, 本文提出假设 5 。

假设 5：政府级别降低时，减弱了企业金字塔层级对企业社会责任的正向作用。

\section{3. 研究设计}

\section{1 变量设计}

3.1.1 被解释变量: 企业社会责任（CSR）。对该变量的衡量本文采用和讯网企业社会责任报 告专业评测体系对企业社会责任的评分。和讯网上市公司社会责任报告专业评测体系从股东 责任、员工责任、供应商、客户和消费者权益责任、环境责任和社会责任五个方面进行考核, 在此基础上设立二级和三级指标, 其数据完整性、评价科学性的特点保证了我们测试的合理 性。

3.1.2. 解释变量:

（1）现金流权（CFR）及两权分离度（SEP）。现金流权为每条控制链持股比例之和, 代表最终控制人参与企业现金分配的权利。两权分离度衡量控制权高出现金流权的部分，可 采用相对数和绝对数两种形式。

（2）金字塔层级数（Layer）。参照 Fan（2003） ${ }^{[20]}$ 和苏坤（2016） ${ }^{[21]}$ 的研究，通过向 上追溯目标企业的控制链条, 在企业采取单一控制途径且终极控制人直接控制上市公司的时 候, 表明不存在中间层公司, 则金字塔层级数取 1 , 依此类推。如果金字塔结构中存在多个 控制链条时, 则以控制层级最长的链条数为依据衡量金字塔层级数。

（3）产权性质（GOV）。产权性质是指当上市公司的实际控制人为国家时，则该上市 公司的性质为国有, 当实际控制人为其他属性的组织时, 则划分为非国有。其他属性的组织 
包括民营企业、社会团体、自然人等。

（4）政府行政级别（Govlevel）。政府行政级别是指国有上市公司实际控制人的行政级 别。本文将行政级别划分为中央政府、省级政府、市级政府以及市级以下四种类型, 当政府 级别为中央时, Govlevel $=1$, 为省级时, Govlevel=2, 为市级时, Govlevel=3, 为市级以下时, Govlevel $=4$ 。

\subsection{3 控制变量}

为了研究终极所有权结构对企业社会责任的影响, 本文选取公司规模、盈利能力、发展 能力、企业价值、股权集中度、行业和年度作为控制变量。其中, 行业分类以证监会《上市 公司行业分类指引》为依据, 排除金融类行业, 获得 19 个行业样本, 并且为消除年度差异对 结果造成的误差, 本文也将年度设置为虚拟变量。

\subsection{4.变量定义}

本文根据研究内容最终设计一个被解释变量、六个解释变量以及七个控制变量，各个变 量的名称、含义以及计算方式如下表所示:

\section{表1 主要变量说明}

\begin{tabular}{|c|c|c|c|}
\hline 变量分组 & 变量名称 & 变量含义 & 计算方式 \\
\hline 被解释变量 & CSR & 企业社会责任 & 和讯网上市公司社会责任报告专业评测体系 \\
\hline \multirow[t]{7}{*}{ 解释变量 } & CFR & 现金流权 & 每条控制链持股比例之和 \\
\hline & SEP & 两权分离度 & 控制权-现金流权 \\
\hline & Layer & 金字塔层级数 & 从终极控制人到目标公司的最多层级 \\
\hline & GOV & 产权性质 & 当企业性质为国有企业是，则 $\mathrm{GOV}=1$, 否则为 0 \\
\hline & Govlevel & 政府行政级别 & 当政府级别为中央时，Govlevel=1；为省级时， \\
\hline & & & Govlevel=2; 为市级时, Govlevel=3; 为市级以下时, \\
\hline & & & Govlevel=4; \\
\hline \multirow[t]{7}{*}{ 控制变量 } & $\mathrm{ROA}$ & 盈利能力 & 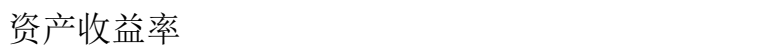 \\
\hline & Size & 公司规模 & 公司总资产的自然对数 \\
\hline & TobinQ & 企业价值 & 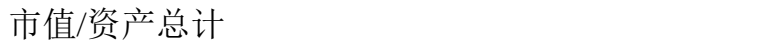 \\
\hline & $\mathrm{OC}$ & 股权集中度 & 公司前十位大股东持股比例之和 \\
\hline & Growth & 发展能力 & 营业收入增长率 ～～～～～～ \\
\hline & $\begin{array}{l}\text { Year }_{n} \\
\text { Industry }\end{array}$ & 年度虚拟变量 & 当公司处于年度 $\mathrm{n}$ 时, 该虚拟变量取 1 , 否则为 0 \\
\hline & & 行业虚拟变量 & 当公司属于 t 行业时, 该虚拟变量取 1 , 否则为 0 \\
\hline
\end{tabular}

\section{2 数据来源}

本文选取中国上交所和深交所 2011-2017 年上市公司为原始数据，并经过以下的筛选:

（1）选择在 2010 年之前上市的公司;（2）剔除金融行业的上市公司。经过上述篮选程序, 最终获得 13389 个有效样本数据, 本文所用有关公司金字塔层级数、终极控制人的行政级别 均由作者手动收集获得, 对于企业社会责任的衡量以和讯网的评分体系为标准, 其他相关数 据来源于 CSMAR 数据库。数据分析使用 STATA15.0 软件进行。

\section{3 模型构建}

本文采用如下模型来检验上述假设:

$$
\begin{aligned}
& C_{S R} R_{i t}=\alpha_{0}+\beta_{1} X_{i t}+\beta_{2} R_{O A A_{i t}}+\beta_{3} \text { Size }_{i t}+\beta_{4} \text { Tobin }_{i t}+\beta_{5} O C_{i t}+\beta_{6} \text { Growth }_{i t}+\beta_{7} \text { year } \\
& +\beta \text { 8industry }+\varepsilon_{i t}
\end{aligned}
$$

其中，CSR 表示企业价值， $\alpha 0$ 表示横截距， $\beta$ 表示回归系数， $\varepsilon$ 表示误差项，X 表示本 文所要研究的解释变量, 具体而言分别为控制权、两权分离度、金字塔层级数、产权性质, 同时将一些影响企业承担社会责任特征变量作为控制变量, 下标 $\mathrm{i}$ 表示第 $\mathrm{i}$ 个样本公司, 小标 $\mathrm{t}$ 表示第 $\mathrm{t}$ 年。

为验证假设 5 , 本文采用以下模型: 


$$
\begin{aligned}
& \text { CSR }_{i t}=\alpha_{0}+\beta_{1} \text { Layer }+\beta_{2} \text { Govlevel }+\beta_{3} \text { Layer }^{*} \text { Govlevel }+\beta_{4}{ }_{2 O A_{i t}}+\beta_{5} \text { Size }_{i t}+\beta_{6} \text { Tobin }_{i t} \\
& +\beta_{7} \text { OC }_{i t}+\beta_{8} \text { Growth }_{i t}+\beta_{9} \text { year }+\beta_{10 \text { industry }}+\varepsilon_{\text {it }}
\end{aligned}
$$

其中, Govlevel 代表政府行政级别，加入交乘项探究政府级别在金字塔层级对企业社会 责任的调节作用, 如果为负, 则假设 5 得到验证。

\section{4. 实证研究结果与分析}

\section{1 描述性统计分析}

将全部样本的主要变量进行描述性统计分析, 结果如表 2 所示。

表 2 列示了相关变量描述性统计分析的结果。从和讯网的企业社会责任评分中可以看出, 评分最高为 90.87 , 最低为-18.23, 其标准差也相对较大, 达到 19.768 , 说明上市公司在企业 社会责任承担方面良莠不齐，仍有较大的发展空间。实际控制人在上市公司中的现金流权最 高可达 $89.99 \%$ ，均值达到 $32.299 \%$ ，股权集中度指标最大值为 $99.187 \%$ ，可见我国上市公司 股权集中度仍然较高。两权分离度指标最大值为 $53.461 \%$ ，均值为 $5.65 \%$ ，表明我国大部分 上市公司都存在着控制权超过现金流权的现象。在金字塔股权结构中, 上市公司金字塔层级 数最多可达到 11 层, 其均值为 2.398 层, 我国上市公司较为普遍的使用金字塔结构进行公司 管理, 且代理关系相对复杂。从收集的样本数据可以看出, 国有上市公司占比 $46.6 \%$, 非国 有上市公司为 $53.4 \%$, 分配相对均衡。总资产报酬率平均为 $0.255 \%$, 盈利能力相对较低, 而 衡量成长性的营业收入增长率平均为 $181.53 \%$ ，说明中国上市公司的成长性较好。对比企业 社会责任评分在全样本中的均值和在国有企业样本中均值可以发现, 国有企业的社会责任评 分较高, 说明国有企业在承担社会责任方面发挥着更显著的作用。

表2 描述性统计结果

\begin{tabular}{ccccccc}
\hline & 变量 & 观测量 & 均值 & 标准差 & 最小值 & 最大值 \\
\hline & CSR & 13389 & 23.743 & 19.768 & -18.23 & 90.87 \\
& CFR & 13389 & 32.299 & 16.85 & 0 & 89.99 \\
& SEP & 13389 & 5.65 & 8.135 & 0 & 53.461 \\
样本量 1 & Layer & 13389 & 2.398 & 1.113 & 1 & 11 \\
(全样本) & GOV & 13389 & 0.466 & 0.499 & 0 & 1 \\
& Size & 13389 & 22.219 & 1.403 & 14.942 & 29.502 \\
& ROA & 13389 & 0.037 & 0.255 & -14.586 & 20.788 \\
& TobinQ & 13389 & 2.368 & 13.034 & 0.007 & 982.982 \\
& OC & 13389 & 55.737 & 15.821 & 3.588 & 99.187 \\
& Growth & 13389 & 4.177 & 181.53 & -11.924 & 20370.57 \\
\hline \multirow{6}{*}{} & CSR & 6268 & 26.271 & 21.876 & -15 & 90.87 \\
样本 2 & CFR & 6597 & 36.579 & 16.789 & 0 & 89.89 \\
(国有上市 & SEP & 6268 & 4.535 & 7.87 & 0 & 43.34 \\
公司) & Layer & 6268 & 2.69 & 1.022 & 1 & 11 \\
& Govlevel & 6268 & 2.027 & .858 & 1 & 4 \\
& Size & 6268 & 22.7 & 1.455 & 16.757 & 28.695 \\
& ROA & 6268 & 0.029 & .115 & -1.558 & 7.249 \\
& TobinQ & 6268 & 1.507 & 2.221 & .007 & 76.093 \\
& OC & 6268 & 56.632 & 15.857 & 12.707 & 99.187 \\
& Growth & 6268 & 2.076 & 46.479 & -4.492 & 3107.432 \\
\hline
\end{tabular}

\section{2 相关性检验}

表格 3 列式了各变量之间的相关系数。企业社会责任承担水平与现金流权、两权分离度、 金字塔层级数、产权性质、公司规模、盈利能力、企业价值、股权集中度均显著相关, 各自 变量之间的相关性都低于 0.5 , 这表明各自变量之间不存在严重的多重共线性。为防止多重共 线性的问题, 本文将逐个引入自变量至模型进行回归分析, 保证研究的可靠性。 
表3 相关性检验

\begin{tabular}{|c|c|c|c|c|c|c|c|c|c|c|}
\hline & CSR & CFR & SEP & Layer & GOV & Size & ROA & TobinQ & Cond & Growth \\
\hline CSR & 1.000 & & & & & & & & & \\
\hline CFR & $0.113^{* * *}$ & 1.000 & & & & & & & & \\
\hline SEP & $0.035 * * *$ & $-0.379 * * *$ & 1.000 & & & & & & & \\
\hline Layer & $0.063^{* * *}$ & $-0.114 * * *$ & $0.358^{* * *}$ & 1.000 & & & & & & \\
\hline GOV & $0.119 * * *$ & $0.237 * * *$ & $-0.128 * * *$ & $0.245^{* * *}$ & 1.000 & & & & & \\
\hline Size & $0.262 * * *$ & $0.242 * * *$ & $0.031 * * *$ & $0.113^{* * *}$ & $0.320 * * *$ & 1.000 & & & & \\
\hline ROA & $0.049 * * *$ & $0.016^{*}$ & 0.007 & -0.011 & $-0.028 * * *$ & 0.005 & 1.000 & & & \\
\hline TobinQ & $-0.034 * * *$ & $-0.046^{* * *}$ & $-0.018^{* *}$ & $0.021 * *$ & $-0.062 * * *$ & $-0.190 * * *$ & -0.008 & 1.000 & & \\
\hline $\mathrm{OC}$ & $0.158^{* * *}$ & $0.619 * * *$ & $0.094 * * *$ & -0.007 & $0.053 * * *$ & $0.295^{* * *}$ & $0.054 * * *$ & $-0.043 * * *$ & 1.000 & \\
\hline Growth & -0.013 & -0.003 & 0.004 & -0.000 & -0.011 & $-0.018 * *$ & 0.001 & 0.001 & 0.004 & 1.000 \\
\hline
\end{tabular}

\section{3 多元回归结果}

模型一和模型二的回归结果在表 4 中展示。

表4 多元回归结果

\begin{tabular}{|c|c|c|c|c|c|c|c|c|}
\hline & \multicolumn{4}{|c|}{ 全样本 } & \multicolumn{4}{|c|}{ 国有上市公司 } \\
\hline & CSR & CSR & CSR & CSR & CSR & CSR & CSR & CSR \\
\hline \multirow{2}{*}{ Constant } & $-72.887 * * *$ & $-72.241 * * *$ & $-72.377 * * *$ & $-70.969 * * *$ & $-77.820 * * *$ & $-78.189 * * *$ & $-80.484 * * *$ & $-84.778 * * *$ \\
\hline & $(-26.818)$ & $(-26.634)$ & $(-26.690)$ & $(-25.554)$ & $(-16.978)$ & $(-17.034)$ & $(-17.439)$ & $(-16.512)$ \\
\hline \multirow{2}{*}{ CFR } & $-0.036 * * *$ & & & & $-0.063 * * *$ & & & \\
\hline & $(-3.317)$ & & & & $(-3.611)$ & & & \\
\hline \multirow{2}{*}{ SEP } & & $0.047 * * *$ & & & & $0.070 * *$ & & \\
\hline & & (2.618) & & & & $(2.422)$ & & \\
\hline \multirow{2}{*}{ Layer } & & & $0.468 * * *$ & & & & $1.059 * * *$ & $2.270 * * *$ \\
\hline & & & $(3.563)$ & & & & $(4.821)$ & $(4.441)$ \\
\hline \multirow{2}{*}{ GOV } & & & & $0.698 * *$ & & & & \\
\hline & & & & $(2.221)$ & & & & \\
\hline \multirow{2}{*}{ Govlevel } & & & & & & & & $1.823^{* *}$ \\
\hline & & & & & & & & $(2.424)$ \\
\hline \multirow{2}{*}{ Layer×Govlevel } & & & & & & & & $-0.705 * * *$ \\
\hline & & & & & & & & $(-2.671)$ \\
\hline \multirow{2}{*}{ Size } & $4.443 * * *$ & $4.411 * * *$ & $4.373 * * *$ & $4.336 * * *$ & $4.549 * * *$ & $4.558 * * *$ & $4.558 * * *$ & $4.594 * * *$ \\
\hline & $(36.662)$ & $(36.451)$ & (35.959) & (34.299) & (22.126) & (22.138) & (22.187) & (22.068) \\
\hline \multirow{2}{*}{ ROA } & $2.837 * * *$ & $2.874 * * *$ & $2.897 * * *$ & $2.926 * * *$ & $51.333 * * *$ & $51.543 * * *$ & $51.877 * * *$ & $51.047 * * *$ \\
\hline & (4.927) & (4.991) & (5.033) & $(5.078)$ & (14.951) & (15.007) & (15.130) & (14.806) \\
\hline \multirow{2}{*}{ TobinQ } & $0.040 * * *$ & $0.041 * * *$ & $0.039 * * *$ & $0.040 * * *$ & $0.606 * * *$ & $0.627 * * *$ & $0.613 * * *$ & $0.622 * * *$ \\
\hline & (3.607) & (3.644) & $(3.439)$ & $(3.617)$ & $(5.229)$ & $(5.409)$ & $(5.300)$ & $(5.366)$ \\
\hline \multirow{2}{*}{$\mathrm{OC}$} & $0.086 * * *$ & $0.061 * * *$ & $0.065 * * *$ & $0.065 * * *$ & $0.076^{* * * *}$ & $0.032 * *$ & $0.033^{* *}$ & $0.033^{* *}$ \\
\hline & (7.274) & $(6.327)$ & $(6.725)$ & $(6.730)$ & (3.850) & (1.973) & (2.024) & $(2.060)$ \\
\hline \multirow{2}{*}{ Growth } & $-0.006^{* *}$ & $-0.006^{* *}$ & $-0.006 * *$ & $-0.006^{* *}$ & $-0.015 * * *$ & $-0.014 * * *$ & $-0.014 * * *$ & $-0.014 * * *$ \\
\hline & $(-2.439)$ & $(-2.382)$ & $(-2.432)$ & $(-2.395)$ & $(-3.098)$ & $(-3.019)$ & $(-2.987)$ & $(-2.989)$ \\
\hline Year & Controlled & Controlled & Controlled & Controlled & Controlled & Controlled & Controlled & Controlled \\
\hline Industry & Controlled & Controlled & Controlled & Controlled & Controlled & Controlled & Controlled & Controlled \\
\hline $\operatorname{Adj}^{2}$ & 0.307 & 0.307 & 0.307 & 0.307 & 0.354 & 0.353 & 0.355 & 0.355 \\
\hline obs & 13,389 & 13,389 & 13,389 & 13,389 & 6,268 & 6,268 & 6,268 & 6,268 \\
\hline
\end{tabular}

（注: * * **, ***分别表示双尾检验在 $10 \%, 5 \%$ 和 $1 \%$ 水平上显著。括号内为 $\mathrm{t}$ 值）

从表 4 的模型 1 中可以看出，现金流权与企业社会责任呈显著负相关关系，即实际控制 人拥有的现金流权越多, 企业承担的社会责任越少, 假设 1 得到验证, 这样表明企业社会责 任是一种代理成本而非 “道德意识”。从模型 2 和模型 3 可以看出, 两权分离度、金字塔层 级与企业社会责任显著正相关, 说明当企业两权分离度越大、金字塔层级数越多的时候, 企 业社会责任成为了掩盖实际控制人对中小股东利益侵占的工具，假设 2 和假设 3 得到验证。 从模型 4 可以看出, 产权性质与企业社会责任显著正相关, 说明相比于非国有企业, 国有企 
业作为政府的形象代言人承担着更多的企业社会责任，假设 4 得到验证。

本文以全样本和国有企业的样本数据为基础, 分别对现金流权、两权分离度、金字塔层 级数与企业社会责任之间的关系进行回归分析, 得到了相同的检验结果了, 也就是说, 在国 有企业中, 同样存在着现金流权与企业社会责任呈显著正相关关系、两权分离度和金字塔层 级数与企业社会责任呈显著负相关关系的现象。

从模型 5 可以看出，在国有企业样本中，金字塔层级数与企业社会责任呈显著正相关关 系，而金字塔层极数和政府级别的交乘项与企业社会责任显著负相关，说明政府级别削弱了 金字塔层级与企业社会责任的正相关程度, 而政府级别越低时, 该数值越大, 意味着削弱的 程度越强，假设 5 得到验证。

同时从表格中可以看出, 本文所选取的控制变量与企业社会责任之间也具有显著相关性。

\section{5. 稳健性检验}

为检验研究结论的稳健性，本文采取以下方法进行了稳健性检验。

1.对变量进行缩尾处理。尽管未进行缩尾的数据已完成了对假设的检验, 但为了降低极 端值产生的不利影响, 本文将连续变量进行了上下 $1 \%$ 的缩尾处理, 采用缩尾后的稳健性检验 回归分析结果仍然支持本文假设。其结果列式如下:

2.变量替换法。变量替换是指对控制变量进行替换，其目的是控制不同变量对实证结果 所产生的差异性。本文逐一对以下控制变量进行了替换：(2)盈利能力的替代, 采用净资产收 益率（ROE）替代资产收益率; (1)公司规模（Size）的替代, 采用期末所有者权益的自然对 数替代公司总资产的自然对数; (3)股权集中度 (OC) 的替代, 采用公司前五大股东持股比例 之和替代公司前十大股东持股比例之和; (4)企业价值（TobinQ）的替代, 采用账面市值比替 代托宾 Q 值; 5发展能力（Growth）的替代, 采用总资产增长率替代营业收入增长率。采用 上述方法逐一替换后的回归结果表明本文的研究结论保持不变。

\section{6. 结 论}

本文从终极所有权结构出发，在理论分析的基础上，以 2011-2017 年上市公司为研究对 象, 深入研究终极所有权结构对企业承担社会责任的影响。研究结论表明：（1）现金流权与 企业社会责任显著负相关, 即随着现金流权的增加, 大股东侵占小股东利益的动机减弱, 减 少了信息不对称带来的冲突，作为代理成本的企业社会责任，其承担意图也会减弱;（2）现 金流权和控制权的分离度与企业社会责任显著正相关, 表明较高的控制权比例增强了控股股 东对上市公司的控制能力以及通过控制权占取私人收益的能力, 为掩盖自身的不当行为, 实 际控制人通过承担企业社会责任的方式转移公众的关注点; （3）金字塔层级数与企业社会责 任显著正相关, 金字塔结构是现金流权与控制权分离的重要原因, 大股东 “掏空一支持一再 掏空一再支持” 的循环过程最终目的是实现 “掏空” ，企业社会责任成了实际控制人谋取私 利行为的遮羞布;（4）国有上市公司比非国有上市公司承担更多的企业社会责任，表明国有 企业经营决策体现国家意志更明显, 相应政府各部门的需要, 履行更多的社会责任;（5）在 国有上市公司中, 其实际控制人的行政级别降低时, 会减弱金字塔层级对企业社会责任的正 向影响, 表明相对于中央等高级别政府, 地方政府控制的上市公司受国家意志的影响较小, 因政治动因承担社会责任的压力减小, 而中央政府面临着更多的社会问题, 其控制的上市公 司将社会效应放在首位。

根据本文的研究结论，本文的政策启示有如下几点：（1）在我国市场化水平不断提高的 过程中, 企业社会责任作为一把 “双刃剑” 需要被合理、适当利用, 在一定情况下也要进行 控制, 防止其成为大股东进行 “掏空” 等不当行为的利器; (2) 为了更好地发挥企业社会责 任的正向影响力，在今后的制度改革中应该鼓励企业自愿披露社会责任并进行相应的政策性 
引导, 让企业社会责任成为企业的主动行为而非出于自身利益考虑的利已主义行为。（3）从 公司自身角度而言, 建立更加完善的利益相关者体系, 权衡各利益相关者之间的关系, 打破 实际控制人一股独大的固有治理模式, 建立共同治理新模式, 在保护利益相关者行使自身权 益的同时，促进公司积极承担更多社会责任。

\section{致谢}

本文为国家自然科学基金项目《组织期望、非正式制度与股价崩盘风险》(71773088)的 阶段性成果之一。

\section{References}

[1] Quan Xiaofeng, Wu Shinong, Yin Hongying. Corporate Social Responsibility and Stock Price Crash Risk: Self-interest Tool or Value Strategy? [J]. Economic Research Journal, 2015,50 (11): 49-64

[2] Derwall J, Koedijk K, Horst J T. A Tale of Values-Driven and Profit-Seeking Social Investors [J]. Journal of Banking and Finance, 2011, 35(8):0-2147.

[3] Hemingway C A, Maclagan P W. Managers' Personal Values as Drivers of Corporate Social Responsibility [J]. Journal of Business Ethics, 2004, 50(1):33-44.

[4] Cheng Xiaoke, Zheng Lidong, Yao Lijie. Does Internal Control Restrict Real Activities Earnings Management?_-A Comparison with Accrual Based Earnings Management [J]. China Soft Science, 2013 (03): 120-131

[5] Shleifer A, Vishny R W. Large Shareholders and Corporate Control [J]. Scholarly Articles, 1986, 94(3):461-488.

[6] La Porta R, Lopez-De-Silanes F, Shleifer A. Corporate Ownership Around the World [J]. The Journal of Finance, 1999, 54(2):471-517.

[7] Shleifer A, Vishny R W . A Survey of Corporate Governance [J]. The Journal of Finance, 1997, 52.

[8] Ingram R W, Chewning E G. The Effect of Financial Disclosure Regulation on Security Market Behavior [J]. Accounting Review, 1983, 58(3):562-580.

[9] Wan Shouyi, Liu Feifei. A Study on the Causes and Economic Consequences of the Lack of Corporate Social Responsibility [J]. Friends of Accounting, 2015 (11): 15-25

[10]Bozec Y, Bozec R. Ownership Concentration and Corporate Governance Practices: Substitution or Expropriation Effects? [J]. Canadian Journal of Administrative Sciences / Revue Canadienne des Sciences de 1 Administration, 2007, 24(3):182-195.

[11]Claessens S, Djankov S, Lang L H P. The separation of ownership and control in East Asian Corporations [J]. Journal of Financial Economics, 2000, 58(1-2):81-112.

[12]Li Chao, Wang Liang. Study on Interest Infringement Behavior of Large Shareholders in Pyramid Share Structure in China [J]. South China Finance, 2012 (1): 57-59

[13]Liu Xing, Dou Wei. Study on Inefficiency Investment in Corporate Based on Private Benefits of Control [J]. Chinese Journal of Management Science, 2009,17(05):156-165.

[14]Lu Yujian. An Empirical Analysis of Earnings Management Behavior of Listed Companies Based on Rights of Allotment [J]. Social Sciences In Nanjing, 2002 (3): 26-32 
[15]Porter M E. Strategy \& Society: The Link Between Competitive Advantage and Corporate Social Responsibility [J]. Harvard Business Review, 2006, 84.

[16]Tian Zhilong, He Yuanqiong. Using Shanyin Marketing to Win the Support of Stakeholders [J]. Marketing Herald, 2005 (04): 52-53

[17]Huang Sujian, Yu Jing. The Nature, Objectives and Social Responsibility of State-owned Enterprises [J]. China Industrial Economy, 2006 (2): 68-76

[18]Zhang Dayong, Fu Liping. Empirical Research on Reduction Behavior of Major Shareholders Based on Ownership Structure and Enterprise Value [J]. Journal of Northwest A\&F University(Social Science Edition), 2010, 10 (3): 56-61

[19] Su Kun. Management Equity Incentive,Risk Taking and Efficiency of Capital Allocation [J]. Journal of Management Science, 2015, 28 (3): 14-25

[20]Fan J, Hanazaki M, Teranishi J. Designing Financial Systems for East Asia and Japan [J]. 2003.

[21]Su Kun. The Effect of State Pyramidal Layers on Corporate Risk Taking_Based on the Difference of Government Control Levels [J]. China Industrial Economics, 2016 (6): 127-143 